\title{
INTERLACING FOR WEIGHTED GRAPHS USING THE NORMALIZED LAPLACIAN*
}

\author{
STEVE BUTLER ${ }^{\dagger}$
}

\begin{abstract}
The problem of relating the eigenvalues of the normalized Laplacian for a weighted graph $G$ and $G-H$, for $H$ a subgraph of $G$ is considered. It is shown that these eigenvalues interlace and that the tightness of the interlacing is dependent on the number of nonisolated vertices of $H$. Weak coverings of a weighted graph are also defined and interlacing results for the normalized Laplacian for such a covering are given. In addition there is a discussion about interlacing for the Laplacian of directed graphs.
\end{abstract}

Key words. Laplacian matrix, Interlacing, Directed Laplacian, Weak coverings.

AMS subject classifications. 05C50, 15A42.

1. Introduction. Given a graph $G$ there are several matrices associated with it. The most commonly studied is the 0-1 adjacency matrix $A$ where $A_{u, v}=1$ if and only if vertex $u$ is adjacent to vertex $v$. Another commonly studied matrix is the combinatorial Laplacian $L=D-A$ where $D$ is the diagonal degree matrix and $A$ is the adjacency matrix. Chung [2] popularized the notion of a normalized Laplacian, defined by, $\mathcal{L}=D^{-1 / 2} L D^{-1 / 2}$ (with the convention that when the degree of $v$ is 0 that $\left.(d(v))^{-1 / 2}=0\right)$.

The normalized Laplacian has gotten increased attention in the last decade due (among other things) to its connections with random walks. Chen et al. [1] (and more recently $\mathrm{Li}[8]$ ) established the following interlacing results for the normalized Laplacian.

THEOREM 1.1. Let $G$ be a simple graph without loops or parallel edges, let $H=e$ be an edge of $G$, and $G-H$ the graph $G$ with edge e removed. If $\lambda_{0} \leq \lambda_{1} \leq \cdots \leq \lambda_{n-1}$ and $\theta_{0} \leq \theta_{1} \leq \cdots \leq \theta_{n-1}$ are the eigenvalues of $\mathcal{L}(G)$ and $\mathcal{L}(G-H)$ respectively, then

$$
\lambda_{k-1} \leq \theta_{k} \leq \lambda_{k+1} \quad \text { for each } k=0,1, \ldots, n-1,
$$

where $\lambda_{-1}=0$ and $\lambda_{n}=2$. More generally if $H$ is a subgraph of $G$ with $|E(H)|=t$ then

$$
\lambda_{k-t} \leq \theta_{k} \leq \lambda_{k+t} \quad \text { for each } k=0,1, \ldots, n-1,
$$

where $\lambda_{-t}=\cdots=\lambda_{-1}=0$ and $\lambda_{n}=\cdots=\lambda_{n+t-1}=2$.

We will establish an improved version of Theorem 1.1. Our first improvement will be to allow a broader range of graphs which include graphs with loops and

*Received by the editors 1 December 2006. Accepted for publication 15 March 2007. Handling Editor: Stephen J. Kirkland.

${ }^{\dagger}$ Dept. of Mathematics, University of California at San Diego, La Jolla, CA 92093-0112, USA (sbutler@math.ucsd.edu). This paper was partially written while the author was visiting the Center for Combinatorics, Nankai University, Tianjin 300071 China. 
multiple edges, more generally any weighted graph. Our second improvement will be to show that when removing a graph from $G$ we can use the number of vertices of the graph being removed rather than the number of edges to control the spread of the eigenvalues. So for instance when we are removing edges from a dense graph such as $K_{10}$ we will show that the eigenvalues spread by at most 10 (the number of vertices), while the above result states that the eigenvalues spread by at most 45 (the number of edges). Before we state the main result we need to introduce terminology needed for weighted graphs.

A weighted graph is a graph (possibly with loops) with a nonnegative weight function $w: V \times V \rightarrow[0, \infty)$ with $w(u, v)=w(v, u)$ (i.e., undirected) and $w(u, v)>0$ if and only if there is an edge joining $u$ and $v$. Using the weight function we define the adjacency matrix by $A_{u, v}=w(u, v)$ and the diagonal degree matrix uses degrees defined by $d(u)=\sum_{v} w(u, v)$. With the adjacency matrix and the diagonal degree matrix we can then define the normalized Laplacian of such a graph as before, i.e., $\mathcal{L}=D^{-1 / 2}(D-A) D^{-1 / 2}$.

A simple graph is the special case when all weights are either 0 or 1 and $w(v, v)=0$ for all $v$. However, by allowing the weights to vary we can model more graphs. For instance, multigraphs can be modeled by letting $w(u, v)$ be the number of edges connecting $u$ to $v$.

Given a weighted graph $G$ we say that $H$ is a subgraph of $G$ if $w_{H}(u, v) \leq w_{G}(u, v)$ for all $u, v$. When $H$ is a subgraph of $G$ we let $G-H$ be the graph which has weight function $w_{G-H}(u, v)=w_{G}(u, v)-w_{H}(u, v)$ for all $u, v$. From this definition it follows that $d_{G-H}(u)=d_{G}(u)-d_{H}(u)$. The graph $G+H$, where the two graphs are on the same set of vertices, is defined analogously. Our main result is as follows.

THEOREM 1.2. Let $G$ be a weighted graph and $H$ a subgraph of $G$ with $t$ nonisolated vertices. If $\lambda_{0} \leq \lambda_{1} \leq \cdots \leq \lambda_{n-1}$ and $\theta_{0} \leq \theta_{1} \leq \cdots \leq \theta_{n-1}$ are the eigenvalues of $\mathcal{L}(G)$ and $\mathcal{L}(G-H)$ respectively, then for $k=0,1, \ldots, n-1$ we have

$$
\lambda_{k-t+1} \leq \theta_{k} \leq \begin{cases}\lambda_{k+t-1} & H \text { is bipartite } \\ \lambda_{k+t} & \text { otherwise }\end{cases}
$$

where $\lambda_{-t+1}=\cdots=\lambda_{-1}=0$ and $\lambda_{n}=\cdots=\lambda_{n+t-1}=2$.

In the proof for Theorem 1.2 we will see that when $H$ is bipartite that we are (essentially) allowed one more degree of freedom than when $H$ is not bipartite. This accounts for the difference in the bounds given by the theorem. The statement of the theorem is the best possible; to see this consider the two graphs shown in Figure 1.1. The graph $G$ has three loops of weight 1 on the three top vertices, three edges of weight 2 (as marked) and the remaining edges of weight 1 . The graph $H$ consists of a triangle of the three top vertices with edge weight 1 along with the loops (so $t=3$ ). Calculating the eigenvalues we have that $\theta_{1}(G-H)=5 / 4>8 / 7=\lambda_{3}(G)$, showing that the bound for non-bipartite graphs cannot be improved in general.

As an example of an application of Theorem 1.2 we have the following.

Proposition 1.3. If $G$ is a simple graph and more than $n / 2$ of the vertices are connected to every other vertex then $n /(n-1)$ is an eigenvalue of $G$.

This follows from the above theorem since $G=K_{n}-H$ where $H$ has fewer than $n / 2$ nonisolated vertices while $n /(n-1)$ is an eigenvalue of $K_{n}$ with multiplicity $n-1$. 

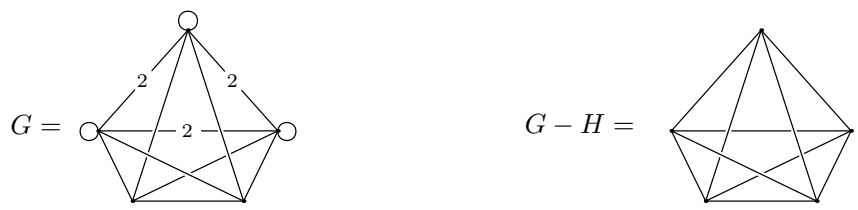

FIG. 1.1. An example showing the result of Theorem 1.2 is tight.

One interesting thing to note is that the result of the theorem is independent of the amount of weight removed. This is because the proof relies on orthogonality conditions which are unaffected by changes in the weights. Instead of subtracting out a graph we could also add a graph. The following result immediately follows from Theorem 1.2 working with the graphs $G+H$ and $(G+H)-H=G$.

COROLlarY 1.4. Let $G$ be a weighted graph and $H$ a graph on the vertices of $G$ with $t$ nonisolated vertices. If $\lambda_{0} \leq \lambda_{1} \leq \cdots \leq \lambda_{n-1}$ and $\theta_{0} \leq \theta_{1} \leq \cdots \leq \theta_{n-1}$ are the eigenvalues of $\mathcal{L}(G)$ and $\mathcal{L}(G+H)$ respectively, then for $k=0,1, \ldots, n-1$ we have

$$
\lambda_{k+t-1} \geq \theta_{k} \geq \begin{cases}\lambda_{k-t+1} & H \text { is bipartite, } \\ \lambda_{k-t} & \text { otherwise }\end{cases}
$$

where $\lambda_{-t}=\cdots=\lambda_{-1}=0$ and $\lambda_{n}=\cdots=\lambda_{n+t-1}=2$.

In Section 2 we will give a proof of Theorem 1.2. In Section 3 we show how to adapt the method of proof of Theorem 1.2 to get an interlacing result for weak covers which we will define. Finally, in Section 4 we examine interlacing for the Laplacian of directed graphs which provided the original motivation for Theorem 1.2.

2. Proof of main result. The proof of Theorem 1.2 will be adapted from the proof of Chen et al. [1] which follows by an application of the Courant-Fischer Theorem (see [7]).

TheOrem 2.1 (Courant-Fischer Theorem). Let $M$ be a real symmetric matrix with eigenvalues $\lambda_{0} \leq \lambda_{1} \leq \cdots \leq \lambda_{n-1}$. Let $\mathcal{X}^{k}$ denote a $k$ dimensional subspace of $\mathbf{R}^{n}$ and $x \perp \mathcal{X}^{k}$ signify that $x \perp y$ for all $y \in \mathcal{X}^{k}$. Then

$$
\lambda_{i}=\min _{\mathcal{X}^{n-i-1}}\left(\max _{x \perp \mathcal{X}^{n-i-1}, x \neq 0} \frac{x^{T} M x}{x^{T} x}\right)=\max _{\mathcal{X}^{i}}\left(\min _{x \perp \mathcal{X}^{i}, x \neq 0} \frac{x^{T} M x}{x^{T} x}\right) .
$$

We will also use the notation $x \perp \mathcal{Z}$ for a set of vectors $\mathcal{Z}$ to indicate that $x \perp z$ for all $z \in \mathcal{Z}$. This is equivalent to saying that $x \perp \operatorname{span}(\mathcal{Z})$ (the span of the vectors of $\mathcal{Z}$ ).

In order to be able to use the Courant-Fischer Theorem we first note that

$$
y^{T}(D-A) y=\sum_{u} y_{u}^{2} d(u)-2 \sum_{u \sim v} y_{u} y_{v} w(u, v)=\sum_{u \sim v}\left(y_{u}-y_{v}\right)^{2} w(u, v) .
$$

If we make the substitution $x=D^{1 / 2} y$, then we have

$$
\frac{x^{T} \mathcal{L} x}{x^{T} x}=\frac{\left(D^{1 / 2} y\right)^{T} \mathcal{L}\left(D^{1 / 2} y\right)}{\left(D^{1 / 2} y\right)^{T}\left(D^{1 / 2} y\right)}=\frac{y^{T} L y}{y^{T} D y}=\frac{\sum_{u \sim v}\left(y_{u}-y_{v}\right)^{2} w(u, v)}{\sum_{u} y_{u}^{2} d(u)} .
$$


REMARK 2.2. Here we run into a small problem when there are isolated vertices, in which case there might be no $y$ for which $x=D^{1 / 2} y$. We will address this in the proof of Theorem 1.2 below, but for now will assume there are no isolated vertices in the graph.

We now have

$$
\begin{aligned}
\lambda_{i} & =\min _{\mathcal{X}^{n-i-1}}\left(\max _{x \perp \mathcal{X}^{n-i-1}, x \neq 0} \frac{x^{T} \mathcal{L} x}{x^{T} x}\right)=\min _{\mathcal{X}^{n-i-1}}\left(\max _{D^{1 / 2} y \perp \mathcal{X}^{n-i-1}, D^{1 / 2} y \neq 0} \frac{y^{T} L y}{y^{T} D y}\right) \\
2) & =\min _{\mathcal{Y}^{n-i-1}}\left(\max _{y \perp \mathcal{Y}^{n-i-1}, y \neq 0} \frac{\sum_{u \sim v}\left(y_{u}-y_{v}\right)^{2} w(u, v)}{\sum_{u} y_{u}^{2} d(u)}\right),
\end{aligned}
$$

and similarly

$$
\lambda_{i}=\max _{\mathcal{Y}^{i}}\left(\min _{y \perp \mathcal{Y}^{i}, y \neq 0} \frac{\sum_{u \sim v}\left(y_{u}-y_{v}\right)^{2} w(u, v)}{\sum_{u} y_{u}^{2} d(u)}\right) .
$$

We are now ready to give the proof for Theorem 1.2 .

Proof. Without loss of generality we may assume that the graph $G$ has no isolated vertices. We first will consider the case when $G-H$ also has no isolated vertices. Since the eigenvalues of the normalized Laplacian always lie in the interval between 0 and 2 inclusive (see [2]), the lower bound for $\theta_{k}$ trivially holds for $k \leq t-1$ while the upper bound trivially holds for $k \geq n-t-1$ ( $k \geq n-t$ if $H$ is bipartite). So for the lower bound we may assume $k>t-1$ while for the upper bound we may assume $k<n-t-1$ (or $n-t$ for $H$ bipartite).

We now show that $\theta_{k} \geq \lambda_{k-t+1}$. Suppose that $\left\{u_{1}, u_{2}, \ldots, u_{t}\right\}$ are the nonisolated vertices of $H$, and let $\mathcal{Z}=\left\{e_{u_{1}}-e_{u_{2}}, e_{u_{1}}-e_{u_{3}}, \ldots, e_{u_{1}}-e_{u_{t}}\right\}$. Then using (2.2) we have

$$
\begin{aligned}
& \theta_{k}=\min _{\mathcal{Y}^{n-k-1}}\left(\max _{y \perp \mathcal{Y}^{n-k-1}, y \neq 0} \frac{\sum_{u \sim v}\left(y_{u}-y_{v}\right)^{2} w_{G-H}(u, v)}{\sum_{u} y_{u}^{2} d_{G-H}(u)}\right) \\
& =\min _{\mathcal{Y}^{n-k-1}}\left(\max _{y \perp \mathcal{Y}^{n-k-1}, y \neq 0} \frac{\sum_{u \sim v}\left(y_{u}-y_{v}\right)^{2} w_{G}(u, v)-\sum_{u \sim v}\left(y_{u}-y_{v}\right)^{2} w_{H}(u, v)}{\sum_{u} y_{u}^{2} d_{G}(u)-\sum_{u} y_{u}^{2} d_{H}(u)}\right) \\
& \geq \min _{\mathcal{Y}^{n-k-1}}\left(\max _{y \perp \mathcal{Y}^{n-k-1}, y \perp \mathcal{Z}, y \neq 0} \frac{\sum_{u \sim v}\left(y_{u}-y_{v}\right)^{2} w_{G}(u, v)}{\sum_{u} y_{u}^{2} d_{G}(u)-\sum_{u} y_{u}^{2} d_{H}(u)}\right) \\
& \geq \min _{\mathcal{Y}^{n-k-1}}\left(\max _{y \perp \mathcal{Y}^{n-k-1}, y \perp \mathcal{Z}, y \neq 0} \frac{\sum_{u \sim v}\left(y_{u}-y_{v}\right)^{2} w_{G}(u, v)}{\sum_{u} y_{u}^{2} d_{G}(u)}\right) \\
& \geq \min _{\mathcal{Y}^{n-k+t-2}}\left(\max _{y \perp \mathcal{Y}^{n-k+t-2}, y \neq 0} \frac{\sum_{u \sim v}\left(y_{u}-y_{v}\right)^{2} w_{G}(u, v)}{\sum_{u} y_{u}^{2} d_{G}(u)}\right)=\lambda_{k-t+1} \text {. }
\end{aligned}
$$

In going from the second to the third line we added the condition that $y$ also be perpendicular to $\mathcal{Z}$ so that we are maximizing over a smaller set. With the condition that $y \perp \mathcal{Z}$ then $y_{u}=y_{v}$ for all $u, v$ in $H$, in particular the second term in the numerator drops out. While in going from the fourth to the fifth line we consider a broader optimization that would include the fourth line as a case. 
Next we show that $\theta_{k} \leq \lambda_{k+t}$ for general $H$. Suppose that $u_{1}, \ldots, u_{t}$ are the nonisolated vertices of $H$, and let $\mathcal{Z}=\left\{e_{u_{1}}, e_{u_{2}}, \ldots, e_{u_{t}}\right\}$. Then using (2.3) we have

$$
\begin{aligned}
\theta_{k} & =\max _{\mathcal{Y}^{k}}\left(\min _{y \perp \mathcal{Y}^{k}, y \neq 0} \frac{\sum_{u \sim v}\left(y_{u}-y_{v}\right)^{2} w_{G}(u, v)-\sum_{u \sim v}\left(y_{u}-y_{v}\right)^{2} w_{H}(u, v)}{\sum_{u} y_{u}^{2} d_{G}(u)-\sum_{u} y_{u}^{2} d_{H}(u)}\right) \\
& \leq \max _{\mathcal{Y}^{k}}\left(\min _{y \perp \mathcal{Y}^{k}, y \perp \mathcal{Z}, y \neq 0} \frac{\sum_{u \sim v}\left(y_{u}-y_{v}\right)^{2} w_{G}(u, v)}{\sum_{u} y_{u}^{2} d_{G}(u)}\right) \\
& \leq \max _{\mathcal{Y}^{k+t}}\left(\min _{y \perp \mathcal{Y}^{k+t}, y \neq 0} \frac{\sum_{u \sim v}\left(y_{u}-y_{v}\right)^{2} w_{G}(u, v)}{\sum_{u} y_{u}^{2} d_{G}(u)}\right)=\lambda_{k+t} .
\end{aligned}
$$

In going from the first to the second line we added the condition that $y$ also be perpendicular to $\mathcal{Z}$ so that we are minimizing over a smaller set. With the condition that $y \perp \mathcal{Z}$ then $y_{u}=0$ for all $u$ in $H$, in particular the second terms in the numerator and denominator drop out. Finally, we consider a broader optimization that would include the second line as a case.

For the case when $H$ bipartite let $\left\{u_{1}, v_{1}\right\},\left\{u_{2}, v_{2}\right\}, \ldots,\left\{u_{t-1}, v_{t-1}\right\}$ be edges of a spanning subgraph of $H$, and let $\mathcal{Z}=\left\{e_{u_{1}}+e_{v_{1}}, e_{u_{2}}+e_{v_{2}}, \ldots, e_{u_{t-1}}+e_{v_{t-1}}\right\}$. Note that if $y \perp \mathcal{Z}$ then for some $\gamma, y_{u}= \pm \gamma$, and in particular $\left(y_{u}-y_{v}\right)^{2}=4 \gamma^{2}$ for all edges $\{u, v\}$ in $H$ (here we are using that $H$ is bipartite). So again using (2.3) we have

$$
\begin{aligned}
\theta_{k} & =\max _{\mathcal{Y}^{k}}\left(\min _{y \perp \mathcal{Y}^{k}, y \neq 0} \frac{\sum_{u \sim v}\left(y_{u}-y_{v}\right)^{2} w_{G}(u, v)-\sum_{u \sim v}\left(y_{u}-y_{v}\right)^{2} w_{H}(u, v)}{\sum_{u} y_{u}^{2} d_{G}(u)-\sum_{u} y_{u}^{2} d_{H}(u)}\right) \\
& \leq \max _{\mathcal{Y}^{k}}\left(\min _{y \perp \mathcal{Y}^{k}, y \perp \mathcal{Z}, y \neq 0} \frac{\sum_{u \sim v}\left(y_{u}-y_{v}\right)^{2} w_{G}(u, v)-2 \gamma^{2} \sum_{u} d_{H}(u)}{\sum_{u} y_{u}^{2} d_{G}(u)-\gamma^{2} \sum_{u} d_{H}(u)}\right) \\
& \leq \max _{\mathcal{Y}^{k}}\left(\min _{y \perp \mathcal{Y}^{k}, y \perp \mathcal{Z}, y \neq 0} \frac{\sum_{u \sim v}\left(y_{u}-y_{v}\right)^{2} w_{G}(u, v)}{\sum_{u} y_{u}^{2} d_{G}(u)}\right) \\
& \leq \max _{\mathcal{Y}^{k+t-1}}\left(\min _{y \perp \mathcal{Y}^{k+t-1}, y \neq 0} \frac{\sum_{u \sim v}\left(y_{u}-y_{v}\right)^{2} w_{G}(u, v)}{\sum_{u} y_{u}^{2} d_{G}(u)}\right)=\lambda_{k+t-1} .
\end{aligned}
$$

We went from the first to the second line as before using the above comments on $\gamma$. In going from the second to the third line we used the following easily proved fact: let $a, b, c$ be real with $2 b \geq a \geq 2 c \geq 0$ and $b>c \geq 0$, then $(a-2 c) /(b-c) \leq a / b$. That the assumptions on $a, b, c$ are satisfied can be easily verified. Finally, we consider a broader optimization that would include the third line as a case.

We now turn to the case when $G-H$ has isolated vertices $u_{1}, u_{2}, \ldots, u_{m}$. We approach this by considering the graph $G_{\epsilon}$ which has loops of weight $\epsilon$ added to the vertices $u_{1}, u_{2}, \ldots, u_{m}$. A simple calculation shows that $\mathcal{L}\left(G_{\epsilon}-H\right)=\mathcal{L}(G-H)$, and in particular has the same eigenvalues, but now with the added loops has no isolated vertices. If we let $\lambda_{k}^{\epsilon}$ denote the $k$ th eigenvalue of $\mathcal{L}\left(G_{\epsilon}\right)$, then the above derivation shows that

$$
\lambda_{k-t+1}^{\epsilon} \leq \theta_{k} \leq \begin{cases}\lambda_{k+t-1}^{\epsilon} & H \text { is bipartite } \\ \lambda_{k+t}^{\epsilon} & \text { otherwise. }\end{cases}
$$

We now let $\epsilon \rightarrow 0$, since $\mathcal{L}\left(G_{\epsilon}\right) \rightarrow \mathcal{L}(G)$ then $\lambda_{k}^{\epsilon} \rightarrow \lambda_{k}$, and the result follows. 
3. Weak coverings and eigenvalues. The technique used to prove Theorem 1.2 can be used to prove other results. In this section we give an interlacing result for weak coverings. We say that $G$ is a weak cover of $H$ if there is some onto mapping $\pi: V(G) \rightarrow V(H)$ such that for all $u, v \in V(H)$,

$$
w_{H}(u, v)=\sum_{\substack{x \in \pi^{-1}(u) \\ y \in \pi^{-1}(v)}} w_{G}(x, y) .
$$

From this definition it follows that $d_{H}(v)=\sum_{x \in \pi^{-1}(v)} d_{G}(x)$. Alternatively, for a weak covering we group the vertices of $G$ in some manner then collapse the individual groups of vertices into single vertices of $H$. To find the edge weights of $H$ we add the weights of any resulting parallel edges that are formed.

REMARK 3.1. The idea of coverings for weighted graphs was previously considered by Chung and Yau [5]. In their definition of a cover they required additional structure which allows eigenvalues from $H$ to be "lifted" up to $G$. Here we make fewer assumptions and so might no longer have eigenvalues lifting up (hence we give the name "weak cover").

THEOREM 3.2. Let $G$ be a weak cover of $H$ with $|V(G)|=n$ and $|V(H)|=m$, and further let $\lambda_{0} \leq \lambda_{1} \leq \cdots \leq \lambda_{n-1}$ and $\theta_{0} \leq \theta_{1} \leq \cdots \leq \theta_{m-1}$ be the eigenvalues of $\mathcal{L}(G)$ and $\mathcal{L}(H)$ respectively. Then for $k=0,1, \ldots, m-1$ we have the following

$$
\lambda_{k} \leq \theta_{k} \leq \lambda_{k+(n-m)} \text {. }
$$

Proof. For $i=1, \ldots, m$ let $V_{i}=\pi^{-1}\left(v_{i}\right)$, i.e., these are the groupings of the vertices of $G$, and let $\mathcal{Z}_{i}=\left\{e_{i_{1}}-e_{i_{2}}, e_{i_{1}}-e_{i_{3}}, \ldots, e_{i_{1}}-e_{i_{j}}\right\}$ where $V_{i}=\left\{v_{i_{1}}, v_{i_{2}}, \ldots, v_{i_{j}}\right\} \subseteq$ $V(G)$. Further we will let $\mathcal{Z}=\bigcup_{i} \mathcal{Z}_{i}$. It is easy to check that the dimension of the span of $\mathcal{Z}$ is $n-m$. Now using (2.3) we have

$$
\begin{aligned}
\theta_{k} & =\max _{\mathcal{Y}^{k} \subseteq \mathbf{R}^{m}}\left(\min _{y \perp \mathcal{Y}^{k}, y \neq 0} \frac{\sum_{u \sim v}\left(y_{u}-y_{v}\right)^{2} w_{H}(u, v)}{\sum_{u} y_{u}^{2} d_{H}(u)}\right) \\
& =\max _{\mathcal{Y}^{k} \subseteq \mathbf{R}^{n}}\left(\min _{y \perp \mathcal{Y}^{k}, y \perp \mathcal{Z}, y \neq 0} \frac{\sum_{u \sim v}\left(y_{u}-y_{v}\right)^{2} w_{G}(u, v)}{\sum_{u} y_{u}^{2} d_{G}(u)}\right) .
\end{aligned}
$$

In the second step we used the defining property of weak covers to lift vectors from $H$ to $G$ so that we still satisfy the same Rayleigh quotient. Our only condition in lifting is that $y_{i}=y_{\pi(i)}$; in particular if $\pi\left(v_{i}\right)=\pi\left(v_{j}\right)$, then we need $y_{\pi(i)}=y_{\pi(j)}$. This last condition is easily achieved by requiring that the lifted vector be perpendicular to $\mathcal{Z}$.

We now bound (3.1) in two ways. First, we can drop the requirement that we remain perpendicular to $\mathcal{Z}$, thus we are minimizing over a larger set and so we have

$$
\theta_{k} \geq \max _{\mathcal{Y}^{k}}\left(\min _{y \perp \mathcal{Y}^{k}, y \neq 0} \frac{\sum_{u \sim v}\left(y_{u}-y_{v}\right)^{2} w_{G}(u, v)}{\sum_{u} y_{u}^{2} d_{G}(u)}\right)=\lambda_{k} .
$$

The second approach is to maximize over some larger set that will also consider the case given in (3.1), i.e.,

$$
\theta_{k} \leq \max _{\mathcal{Y}^{k+n-m}}\left(\min _{y \perp \mathcal{Y}^{k+n-m}, y \neq 0} \frac{\sum_{u \sim v}\left(y_{u}-y_{v}\right)^{2} w_{G}(u, v)}{\sum_{u} y_{u}^{2} d_{G}(u)}\right)=\lambda_{k+n-m}
$$


Combining the two inequalities above concludes the proof.

Alternatively it is easy to show that Theorem 3.2 follows from a result of Haemers [6] on interlacing of eigenvalues of matrices. We have given the proof above to emphasize the nature of the approach.

4. Comments on interlacing for directed graphs. Recently Chung [3] defined a Laplacian for aperiodic strongly connected directed graphs and showed connections of its spectrum to mixing rates of random walks and isoperimetric properties, and in a subsequent paper gave a further connection to the diameter of the graph [4]. A natural question is whether a similar interlacing result to Theorem 1.2 holds for the Laplacian of a directed graph. In this section we will partially answer this question in the negative.

Definition for the directed Laplacian. To define the Laplacian for a directed graph $\vec{G}$ we start with $P$ the probability transition matrix with $P_{u, v}$ the probability of moving from $u$ to $v$ (for a weighted directed graph $P_{u, v}=w(u, v) / d_{\text {out }}(u$ ) where $d_{\text {out }}(u)=\sum_{t} w(u, t)$ is the out-degree of $\left.u\right)$. If we let $\mathbf{1}$ denote the all 1 s vector then $P \mathbf{1}=\mathbf{1}$. If we assume that the graph is strongly connected and aperiodic it follows from the Perron-Frobenius Theorem (see [7]) that there is a unique (row) vector $\phi$ for which $\phi P=\phi$ with $\phi(v)>0$ for all $v$ and $\sum_{v} \phi(v)=1$. This vector $\phi$ is called the Perron vector of $P$.

If $\Phi$ is the diagonal matrix with $\Phi(v, v)=\phi(v)$, then the directed Laplacian is defined by Chung [3] as

$$
\mathcal{L}(\vec{G})=I-\frac{1}{2}\left(\Phi^{1 / 2} P \Phi^{-1 / 2}+\Phi^{-1 / 2} P^{*} \Phi^{1 / 2}\right),
$$

where $P^{*}$ denotes the transpose of $P$.

With this definition it is not too difficult to construct counterexamples to the corresponding statement of Theorem 1.1 for directed graphs. For example if we consider the simple directed graphs given in Figure 4.1 we have that $\lambda_{2}(\vec{G})=0.324609 \ldots<$ $0.362281 \ldots=\lambda_{1}(\vec{G}-\vec{H})$.
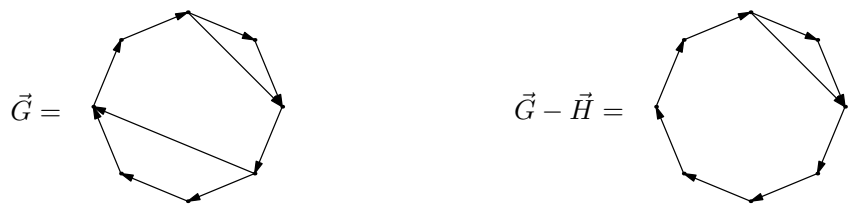

FIG. 4.1. An example of a directed graph where the eigenvalues do not interlace.

Connecting directed Laplacians with undirected Laplacians. To understand why the corresponding statement of Theorem 1.1 could fail for directed graphs we connect the Laplacian for a directed graph with the Laplacian for a corresponding undirected graph.

LEMMA 4.1. Let $\vec{G}$ be an aperiodic strongly connected weighted directed graph and let $H$ be a weighted undirected graph on the same vertex set with weights defined 
by

$$
w(u, v)=\phi(u) P(u, v)+\phi(v) P(v, u) .
$$

Then $\mathcal{L}(\vec{G})=\mathcal{L}(H)$.

Proof. We note first that since $\phi$ is a right eigenvector of $P$ and $\sum_{v} P(u, v)=1$, it follows that $d_{H}(v)=\sum_{v} w(v, t)=2 \phi(v)$.

For terms on the diagonal we have

$$
\mathcal{L}(\vec{G})_{v, v}=1-P(v, v)=1-\frac{w(v, v)}{2 \phi(v)}=1-\frac{w(v, v)}{d_{H}(v)}=\mathcal{L}(H)_{v, v},
$$

while for the off-diagonal terms

$$
\begin{aligned}
\mathcal{L}(\vec{G})_{u, v} & =-\frac{1}{2}\left(\sqrt{\frac{\phi(u)}{\phi(v)}} P(u, v)+\sqrt{\frac{\phi(v)}{\phi(u)}} P(v, u)\right) \\
& =-\frac{(\phi(u) P(u, v)+\phi(v) P(v, u))}{\sqrt{(2 \phi(u))(2 \phi(v))}}=-\frac{w(u, v)}{\sqrt{d_{H}(u) d_{H}(v)}}=\mathcal{L}(H)_{u, v} .
\end{aligned}
$$

Establishing the equality.

REMARK 4.2.

- This connection between directed and undirected Laplacians can be used to establish several results. For instance it can be shown that the Cheeger inequality established for the directed graph $\vec{G}$ in Chung [3] is equivalent to the already known Cheeger inequality for the undirected graph $H$ defined in Lemma 4.1.

- The underlying principle of the directed Laplacian is based on circulations, i.e., a nonnegative function $F: V \times V \rightarrow[0, \infty)$ with the property that at each vertex $u$

$$
\sum_{v} F(v, u)=\sum_{t} F(u, t) .
$$

If we think of the circulation $F$ as a flow then the above equality can be interpreted as saying that at each vertex the in-flow equals the out-flow. Chung [3] showed that $F(u, v)=\phi(u) P(u, v)$ is a circulation and uses this to establish the directed Laplacian. It is easy to adapt Lemma 4.1 to give new definitions for directed Laplacians using different types of circulations, and then establish some corresponding Cheeger inequalities and other similar results.

Applying Lemma 4.1 to the graphs in Figure 4.1 we get the weighted undirected graphs shown in Figure 4.2, where unspecified edges have weight 1. [We have scaled the weights in Figure 4.2 to more easily compare the two graphs; it is simple to see by (2.1) that scaling all the weights by some constant factor does not change the spectrum.]

Now we see that the removal of a single edge in the directed graph in Figure 4.1 had an effect on many edges in the underlying undirected graph in Figure 4.2. So by 

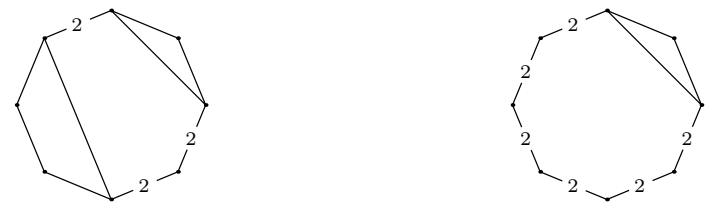

FIG. 4.2. The corresponding undirected graphs for graphs given in Figure 4.1.

Theorem 1.2 the eigenvalues could spread by more than just 1 , and in our case could spread by at most 3 .

In general the removal of a single edge in a directed graph can have a tremendous impact on the underlying undirected graph. Using the results of Theorem 1.2 for the underlying undirected graph then we cannot in general guarantee a tight spread of the eigenvalues between the two graphs. Although for some special cases it can be shown that the removal of a single edge has a small impact on the underlying undirected graph in which case we can get a similar result.

This does not conclusively say that there is no corresponding statement such as Theorem 1.1, but only illustrates the difficulty of using the approach given by use of the Courant-Fischer Theorem. It would be interesting to see if there were some construction such that for each $k$ there exists an aperiodic strongly connected directed graph $\vec{G}$ such that the removal of a single edge leaves an aperiodic strongly connected directed graph $\vec{G}-\vec{H}$ and either $\lambda_{m+k}(\vec{G})<\lambda_{m}(\vec{G}-\vec{H})$ or $\lambda_{m-k}(\vec{G})>\lambda_{m}(\vec{G}-\vec{H})$ for some $m$.

Acknowledgment. The author thanks Fan Chung for support and enlightening discussions on the subject of normalized Laplacians and to the referees whose suggestions helped improve the quality of the paper. The author also expresses his appreciation to the Center for Combinatorics at Nankai University for their support and enthusiastic reception on the topic of spectral graph theory.

\section{REFERENCES}

[1] G. Chen, G. Davis, F. Hall, Z. Li, K. Patel, and M. Stewart. An interlacing result on normalized Laplacians. SIAM Journal on Discrete Mathematics, 18:353-361, 2004.

[2] F. Chung. Spectral Graph Theory. CBMS Regional Conference Series in Mathematics 92. AMS, Providence, RI, 1997.

[3] F. Chung. Laplacians and the Cheeger inequality for directed graphs. Annals of Combinatorics, 9:1-19, 2005.

[4] F. Chung. The diameter and Laplacian eigenvalues of directed graphs. Electronic Journal of Combinatorics, 13:N4 (6 pp.), 2006.

[5] F. Chung and S.-T. Yau. Coverings, heat kernels and spanning trees. Electronic Journal of Combinatorics, 6:R12 (21 pp.), 1999.

[6] W. H. Haemers. Interlacing eigenvalues and graphs. Linear Algebra and its Applications, 226228:593-616, 1995.

[7] R. A. Horn and C. R. Johnson. Matrix Analysis. Cambridge University Press, Cambridge, 1990.

[8] C.-K. Li. A short proof of interlacing inequalities on normalized Laplacians. Linear Algebra and its Applications, 414:425-427, 2006. 